\title{
What is Addictive in Internet?
}

\author{
Gabriel Thorens*, Alexandre Wullschleger, Riaz Khan, Sophia Achab and Daniele Fabio Zullino
}

Division of Addictology, University Hospitals of Geneva, Switzerland

\begin{abstract}
Focusing on World of Warcraft (WoW), the most popular massive multiplayer online role playing game (MMORPG), the present article stresses, that, similarly to addictive drugs, certain features of Internet applications may act on the mesolimbic dopaminergic system, and thus become addictive.

The distinction is made between addictive products (e.g. nicotine, specific stimuli), first order vectors (e.g. tobacco, WoW software) and second order vectors (e.g. cigarettes, internet technology).

Stimuli presented by Internet contents are described which have been reported in other contexts to induce addiction related neurobiological activities, i.e. stimuli with reference to sexuality, social acceptance, fairness, cooperative social interactions, social hierarchies, and humor.

The mode of presentation of these stimuli represents another important issue. The development of WoW can be described as largely based on operant conditioning aspects, sequencing continuous and intermittent reinforcement, fixed- and variable-ratio schedules, purposely to initiate and maintain repetitive behavior.

Regarding their addictive potential, the properties of the Internet technology can be discussed regarding two aspects: (a) accessibility/facility of use, and (b) rapidity of action. The addictive properties of WoW have probably been facilitated by the last technological developments, rendering the game more accessible, and permitting very strong time correlations between potentially addictive stimuli and the reinforced cognitions and behaviors.
\end{abstract}

Keywords: Internet addiction, Video game, World of Warcraft, Reinforcement.

\section{INTRODUCTION}

It has repeatedly been asserted that Internet addiction is a sub-category of addictive behaviors sharing the clinical and neurobiological characteristics which can define addiction as a general clinical category. Thus, finding similarities between the examined new entity (Internet addiction) and those already established (e.g. nicotine addiction) may be a momentous element to corroborate the claim. This paper will review available data on certain features of Internet applications which are supposed to act on the same neurobiological structures involved in substance-related addictions, e.g. the mesolimbic dopaminergic system.

In doing so, the focus will be on World of Warcraft (WoW), the most popular massive multiplayer online role playing game (MMORPG) worldwide. At the end of 2008, WoW was reaching 11 millions players. The last extension of the game (Wrath of the Leech King) was sold at 2.8 million copies within the first 24 hours of availability.

\section{DESCRIPTION OF WORLD OF WARCRAFT}

Within WoW, players buy or download the game and get a number of free trial days, then they have to pay a monthly fee of approximately twenty US dollars (depending on the country in which they bought the game). At the beginning,

*Address correspondence to this author at the Division of Addictology, University Hospitals of Geneva, Rue Grand-Pré 7OC, 1202 Genève Switzerland; Tel: + 412237257 50; Fax: + 412237257 54;

E-mail: Gabriel.Thorens@hcuge.ch players get a game account and choose a specific server. Each server contains thousands of players and is PvE (player $v s$ environments: the players are fighting together against the non player characters), PvP (player $v s$ player) or RP (role play, where players are supposed to chat and act as if they incarnate their character in the game) oriented. Servers are regrouped by languages.

Players pick a character between two factions: horde or alliance. They choose a race (human, troll, gnome...) and an ability (wizard, priest, warrior...). Everybody starts at level 1 with the same chance to progress through the game. Depending on their abilities, players will be assigned to one of three specific roles: healing, making damages and tanking (absorbing damages). Until level 80 (the last level a player can reach with the last extension), the game is a succession of repetitive quests based on a variation of: killing $X$ monsters, bringing $\mathrm{Y}$ objects to character $\mathrm{Z}$. The more the player progresses in the levels, the more he will be able to join other players for group quests, group instances (specific dungeons for 5 players), raids (dungeons for 10 or 25 players) or player $v s$ player fights such as arena tournaments. Before level 80, players cannot participate to arena tournaments or specific raids. At any time players can join a guild, i.e. a group with a structured hierarchy (guild leader, officers, members and rookies) designed to enhance progression, share competences and, at level 80 , to organize specific groups for raids. The rapid progression in the game nor only depends on grouping with others in guilds, but mainly on spending an amount of time playing the game. The more one plays, the faster he could reach top levels and 
acquire interesting objects. This could be a facilitating factor to addictive way of playing; such as the attractive social aspect of the game.

\section{DISTINGUISHING VECTORS AND PRODUCTS IN THE FIELD OF ADDICTIONS}

One neglected issues in the discussion of addictive products is the distinction between addictive products and their vectors. We hereafter will first try to clarify the product-vector distinction through the example of nicotine addiction, and subsequently discuss it with regard to internet addiction.

The distinction between addictive product and vector becomes rapidly evident when examining the triad cigarette, tobacco and nicotine. Nicotine, the addictive substance, is a component of tobacco, which itself is most often consumed as cigarette. Thus, the product nicotine is carried by tobacco, which therefore becomes a vector for nicotine. We could call this a vector of first order regarding nicotine. A cigarette, on the other hand, is a mean to carry tobacco. We will thus call it a vector of second order regarding nicotine. This leads to multiple possible combinations between products and vectors. For example, consider that nicotine can be carried by tobacco, by patches, by gums, etc. Tobacco can be smoked by means of cigarettes, cigars, pipes, water pipes, etc. A cigarette can contain tobacco, marijuana, etc.

The addictive effects of smoking a cigarette will depend:

- On the addictive property of nicotine, i.e. from specific effects which will generate addiction (which is subject of pharmacodynamics).

- On the concentration of nicotine in tobacco, i.e. from properties of the vector of first order (which is subject of pharmacokinetics).

- $\quad$ On the design of cigarette, i.e. from properties of the vector of second order (which again is subject of pharmacokinetics).

- On the individual vulnerability of the smoker (which is subject, in part, of pharmacogenetics).

- $\quad$ On the technique of smoking.

The first 3 factors will be the topic of the present paper.

The questions that arise are thus:

- Which are the stimuli within the internet that may possibly induce addiction related brain processes? We will describe stimuli presented by Internet contents which have been described in other contexts to induce addiction related neurobiological activities. Thus, we will try to define the product of internet addiction.

- Which are the properties of Internet applications that promote the addictive efficiency of the products? Thus, we will try to examine the addiction promoting properties of the first order vector, the WoW software.

- Which are the properties of the Internet technology that boost the development of addictive Internet consumption behaviors? Thus, we will try to analyze the addiction promoting properties of the second order vector.

\section{The Products of Internet Addiction}

Some of the most important aspects of learning and expression of motivated behaviors (and addiction in particular), have consistently been found to be related to a network of cerebral components interconnected via glutamatergic fibers, receiving dopaminergic afferents from the ventral tegmental area (VTA) and comprising especially the prefrontal cortex (PFC), the hippocampus, the amygdala and the nucleus accumbens (NAcc) [1]. The activity of the NAcc, a major part of the ventral striatum and a central node within the limbic system, will be the main focus of the present review. NAcc serves as an interface between corticolimbic regions important for motivation and motor regions important for behavioral output and plays a key role in generating motivated behaviors related to natural rewards as well as drugs of abuse. It seems among others to mediate the reinforcing impact of drugs of abuse.

The ventral striatum/nucleus accumbens has been found to be activated in human neuroimaging experiments when a reward is received as well as when a reward is expected [2]. For example, ventral striatal activity correlates with the amount of money a subject can earn in an experiment and thus indicates reward value. The predictability of the reward seems to be the most important information the NAcc carries. It has been proposed that it is not the prediction error but salience in general that is coded by the NAcc [3]. Salience can be defined as the property of a stimulus that is unexpected as well as arousing and elicits an attentionalbehavioral switch. This position is among others sustained by evidence that negatively rated stimuli elicit activation in the NAcc too.

All known addictive drugs have been found to increase the extracellular concentration of dopamine in the nucleus accumbens.

\section{INTERNET STIMULI WHICH ACTIVATE THE STRIATUM}

We will hereafter review different classes of environmental stimuli which have been found to be associated with activations of the ventral striatum, and try to parallel them with specific features and contents of WoW.

Nucleus accumbens dopamine levels have not only been found to be elevated in response to primary rewards such as food delivery or sexual stimuli, but also in response to secondary rewards like monetary incentives [4-6].

While there are no explicit references to sexual content in WoW, players can chose between a male or female character and can customize their appearance. Choosing "sexy" outfits may therefore sexualize the content. The interaction between players through vocal or written chats is a possible way of seductive behavior. Some gamers overuse the in game seduction and may gladly gender swap in order to benefit from some gamers generosity (offering some rare objects) or assistance. The anonymity may also contribute to enhance such seductive social interactions. Furthermore, Internet is most likely known to enhance the intensity of the interpersonal interactions as described by Walthers [7] as "hyperpersonal communication".

The presence of primary rewards is thus probably rather limited. Secondary rewards are, on the other hand, frequent. 
In an interesting PET-study, raclopride binding to dopamine receptors in the striatum was found to be significantly reduced in 8 male volunteers during a video game which involved learning to navigate a tank for a monetary incentive [8].

Humans are living in a highly social environment, in which most accomplishments happen through collaboration with others [9]. Cues that indicate social acceptance or being treated fairly by others may thus be highly salient and potentially induce reinforcement. Several neuroimaging studies have actually reported activation in the striatum areas in response to several social stimuli [10].

It has repeatedly been found that cooperative social interactions do activate the striatum whereas non-cooperative behaviors do not $[2,11]$. Also, such activations have been found when subjects experience mutual cooperation with a human partner compared with mutual cooperation with a computer partner, and this despite the fact that the monetary gain was identical in both situations [12].

Within WoW, the way players interact with other people is very progressive. When starting, there is no need to play with other human characters; players can progress on their own. They are, yet, always surrounded by others they can compare to (there is an option in the game, for looking at their experiences or equipments). It has, besides, been found that during the leveling phases, players tend to progress faster if they play alone [13].

Furthermore, even the "non collaborative" parts of the game, i.e. the "player vs player competition", are actually all designed to integrate important collaborative elements. For example, the so-called "arena fights" are designed to let compete 2 vs 2, 3 vs 3 and 5 vs 5 players, but never 1 vs 1 . Players have to collaborate with at least one other person if they want to engage in such "arena fights". Also, it becomes imperative for ambitious players to affiliate with complementary characters, to counterbalance their own weaknesses. For example, a strong warrior will try to team with a frail priest, a character that has the capacity to heal injuries of the other characters, who will reciprocally be able to heal him.

Human studies have suggested that fairness based cooperation may be even more reinforcing than cooperation in itself. Fair offers e.g. lead to increased activity in the ventral striatum compared with unfair proposals of equal monetary value [9]. In a particularly illustrating study [14] subjects first played a sequential prisoners dilemma game with (confederate) fair and unfair opponents. The focal subjects were then scanned (using fMRI) when a slight pain - an electrical shock - was administered either to themselves or to confederate partners who behaved fairly or unfairly. Both men and women exhibited empathic responses in anterior cingulate and anterior insula when the fair partner received pain. However, only men reported a higher desire for revenge against unfair partners, and also exhibited activation in the NAcc and orbital prefrontal cortex when unfair partners were shocked. Male revenge desire ratings across subjects were also correlated with the estimate of NAcc activity, consistent with the view that there is a reward value in observing the punishment of unfair partners.
Fairness is a central preoccupation for the designers and administrators of WoW. Thus, GMs (game masters, i.e. employees of the game editor who play a mediating role in game) are constantly accessible, answering questions and resolving quarrels between players. As in "real world" human society, specific rules have been edited, forbidding harassment, stealing, etc. transgressing these rules can lead to a definitive ban of a player account. Moreover, the WoW community constantly develops social regulations within the game. For example, a specific WoW term for thieves has been coined: "Ninjas", whose "bad behaviours" are denounced in the general chat channel.

Human social hierarchies are prominent in many different settings (domestic, professional, recreational) where they define implicit expectations and action dispositions that drive suitable social behaviors [15]. Status within a social hierarchy is often made explicit (e.g., via uniforms, honorifics, verbal assignment, or even in some languages via status-specific grammar), but it can also be inferred from cues such as facial features, height, gender, age, and dress. According to Festinger's theory of social comparison processes [16], hierarchical ranks play a significant role in achieving accurate self-knowledge and self-improvement. This is particularly so when subjects make upward social comparisons, i.e., comparisons between oneself and an individual of higher status.

In a recent study using an interactive game, two experiments were used to create a social hierarchy [15]. Brain activity in ventral striatum was significantly greater when subjects viewed a player of a higher rank than a player of a lower rank. In another interesting study, Erk et al., [17] presented different types of grey-scaled photographs of cars (sport cars, middle-sized cars and small cars) to men. They were asked to assess the attractiveness of the cars irrespective of the costs or practical considerations. Sports cars were judged by the respondent's significantly more and small cars significantly less attractive than medium-sized cars. More attractive cars elicited stronger activations in ventral striatum. It has been proposed that sports cars do signal social dominance, as dominance and social rank are mediated by individual attributes signaling wealth and superfluity (the peacock's tail principle).

Not only do social comparisons affect activation levels in the ventral striatum, even if subjects are not actively engaged in decision-making [18] but the acquisition of a good reputation was also found to activate the striatum [10].

The more WoW users play; the more they need to interact with others to progress, i.e. the more they have to compare themselves with their opponents and teammates. Thus, the development of tenaciously competitive guilds can regularly be observed, that are largely oriented in breaking game records and being first in achieving specific game goals. Their hiring criteria are very strict and their rules are nearly martial. Players can access these guilds only if they are ready to play 3 to 5 nights per week and to keep a very competitive level if they do not want to be excluded.

Also, the equipment of characters (armory, weapons etc.) is classed in different categories: common, rare and "epic" (the toughest to get). These equipment classes are rendered easy to spot by other players, as they are marked by different 
colors. For example, all "epic stuffs" are purple. The game also includes a feature permitting with a simple command to check all equipments of each nearby player.

Just as sports cars can signal social dominance by signaling superfluity (the peacock's tail principle), WoW gives the possibility to acquire not only useful objects (in term of improving abilities), but plenty of ostentatious ornamental objects, e.g. a dragon who has the same speed as a normal mount but a much more showy aspect. Furthermore, players can acquire "pets", which are simply "useless" animals following the avatars. Some of these "pets" are especially rare and difficult to acquire.

Finally, humor, another important social interaction feature, has been found to engage the nucleus accumbens [19]. The degree of humor intensity was positively correlated with BOLD (Blood-oxygen-level dependent) signal intensity.

Amusingly, humor is largely present in WoW. E.g. there are various "Easter eggs" integrated within quests, environment, nonplayer characters, etc., to be found by players. They are often humorous references to literature, movies, etc. For example, the character called Haris Pilton sells the biggest and most expensive bag of the game.

\section{The First Order Vector}

While the occurrence of salient stimuli during the game is a necessary causal factor for reinforcement, it is in itself not sufficient. The mode of presentation of these stimuli represents another important issue. The next step will be to question characteristics of the first order vector (i.e. the WoW-software) which are suitable to sustain the reinforcing effects of the stimuli. While nicotine's effect largely depends from its kinetics, the same can be supposed for WoW. Thus, the "kinetics" of the game can be expected to basically influence its addictive properties. "Kinetics" in this context means the pattern of stimulus-presentation.

The model of operant conditioning will be used to examine this issue. Operant conditioning is the process of behavior modification in which the likelihood of a specific behavior is increased or decreased through positive or negative reinforcement each time the behavior is exhibited. Through operant conditioning, an association is made between a behavior and its consequence.

Hence the question arises: how is WoW designed in order to use addictive stimuli in operant conditioning?

Stimuli may be presented in the environment according to two basic categories of schedule: continuous and intermittent.

In continuous reinforcement, the desired behavior is reinforced every single time it occurs. Generally, this schedule is best used during the initial stages of learning in order to create a strong association between behavior and response. Once the response is firmly attached, reinforcement in research is usually switched to a partial reinforcement schedule. When analyzing WoW, it becomes rapidly evident, that both classes of schedules are used in a compound manner, which will be discussed below.

In intermittent (or partial) reinforcement, the response is reinforced only part of the time. The reward is delivered after a determined amount of time or after a determined number of correct responses. These conditions can always stay the same (fixed) or can change over time (variable). Learned behaviors are acquired more slowly with intermittent reinforcement, but the response is more resistant to extinction.

There are four schedules of partial reinforcement, which all differ regarding the elicited response patterns: Fixed-ratio schedules, variable-ratio schedules, fixed-interval schedules, and variable-interval schedules. In $\mathrm{WoW}$, we can find particularly variable-ratio schedules and fixed-interval schedules.

Variable-ratio schedules occur when a response is reinforced after an unpredictable number of responses. This schedule creates a high steady rate of responding. Gambling and lottery games are good examples of variable ratio schedules. Variable-ratio schedules are best for maintaining behavior.

This type of schedule seems to be largely the basis of WoW. Players are, e.g. requested do a repetitive rapid task (killing monsters) to get a potential reward which is always a certain amount of virtual money and sometimes a rare item. This item represents the reinforcer after an unpredictable number of responses $(0.5 \%$ chance to be obtained by killing a monster).

Fixed-interval schedules are those where the first response is rewarded only after a specified amount of time has elapsed. The time period required is always the same.

This schedule causes high amounts of responses near the end of the interval, but much slower responses immediately after the delivery of the reinforcer.

WoW uses fixed-interval schedules too. For example, changing silver into gold is an ability of the profession "alchemists". After having changed one silver ingot into a gold ingot, a fixed time interval begins during which no other conversions are possible. Another example of fixedinterval schedule applied in WoW is the one weekly authorization to compete in specific rewarding instances.

Ratio schedules produce higher rates of responding than interval schedules, when the rates of reinforcement are otherwise similar. Variable schedules produce higher rates and greater resistance to extinction than most fixed schedules. The variable-ratio schedule produces both the highest rate of responding and the greatest resistance to extinction. This also explains gamblers' addictive behaviors at slot machines, which are designed to apply variable-ratio schedules. The same is true for WoW.

\section{COMPOUND SCHEDULES}

Thus WoW seems to use most of the simple reinforcing schedules, it can also combine them to become so-called compound schedules. These combine two or more different simple schedules in some way using the same reinforcer for the same behavior.

Multiple schedules are schedules where anyone of two, or more, schedules may occur with a stimulus indicating which one is in force. For example, a schedule A may be operational when given a red light and a schedule $\mathrm{B}$ when given a yellow light. In WoW, such a change of schedule can 
be found for example in the battle against specific monsters. Some monsters can change their form, switching between two or more shapes and colors. Each form signals a different schedule according to which it can be defeated. In one form, the monster can be vulnerable to a certain amount of sword hits (variable-ratio schedule), and in the alternative form only after a certain time lap (variable-interval schedule).

Concurrent schedules are those, where 2 or more schedules are simultaneously in force though not necessarily on 2 or more different response devices, and reinforcement on those schedules is independent of each other. Thus, they are schedules of reinforcement that are simultaneously available to a subject, so that he can respond on either schedule. Each behavior pays off according to its own schedule of reinforcement. Concurrent schedules often lead to rapid alternation between the keys. One illustrative example from WoW may be the possibility to defeat an enemy through different weapons or magic spells, each method having its own schedule of reinforcement.

Another form of compound schedules is the so called chained schedules, where reinforcement occurs after two or more successive schedules have been completed, with a stimulus indicating when one schedule has been completed and the next has started. Chaining thus involves linking discrete behaviors together in a series, such that each result of each behavior is both the reinforcement (and consequence) for the previous behavior, and the discriminative stimuli (or antecedent) for the next behavior. At the end of the chain, a reinforcer is given. This principle is largely present in WoW. For example, quests are usually chained to attain a specific goal. E.g. the avatar of a player is solicited to acquire a specific metal (obtaining experience points and possibly gold), to carry it to a blacksmith (gaining again rewards), to use the sword manufactured by the blacksmith to combat a monster (gaining rewards), and finally to bring the monster's head to the king (receiving a particularly valuable item). A further striking example is the leveling process itself.

A further interesting form of reinforcement which has been described is the so called differential reinforcement of high rates (DRH). This term describes a schedule of reinforcement in which the reinforcement is provided at the end of a predetermined interval contingent on the number of responses emitted during the interval being greater than a gradually increasing criterion based on the individual's performance in precious intervals. DRH can be used to increase high rates of responding. It is like an interval schedule, except that a minimum number of responses are required in the interval in order to receive reinforcement. One remarkable example from WoW may be the healing activity of healers. Take for example, a healer who is committed to restore health points of a warrior who himself is fighting a monster. As the warrior may continuously be injured by the monster, he has to repeatedly be healed by the healer in order to continue his fight interval condition. It will be the healers' (and the warriors') interest that the healer rapidly and repeatedly casts the healing spells, as they "cool down" within few seconds, i.e. loose their effect. It is also prohibited to automatize these procedures, thus forcing the healer to repeat the action manually.

\section{The Second Order Vector}

Just as cigarettes can be considered as vectors of second order to carry tobacco and nicotine, the internet technology represents a vector of second order to carry the WoW game. The characteristics of second order vectors may be considered regarding two aspects: (a) accessibility/facility of use, and (b) rapidity of action.

Regarding the accessibility/facility of use aspect, commercially manufactured cigarettes today represent the best option to facilitate initiation and maintenance of nicotine use. Similarly, Internet technologies have constantly been developed in order to increase the so-called AAA principle (accessibility, affordability, and anonymity), rendering Internet products increasingly available for a large population. An obvious interest for the game designers is to implement WoW on mobile devices, improving the vector for the same product. In doing so, the salient stimuli present in Wow will remain the same, but the availability of the product will greatly increase. There is already a version of the WoW armory (an online list of avatar's characteristics and statistics) available on some new phones.

Regarding the rapidity of action aspect, the diffusion speed of addictive substances in the brain has been recognized as affecting their reinforcing effects. This association has among others been shown in PET studies that evaluated the pharmacokinetics of cocaine, documenting that it was the fast uptake of the drug into the brain but not the brain concentration per se that was associated with getting high (reviewed in [20]). Thus, faster the dopamine increases, the more intense the reinforcing effects. Speed is also an imperative preoccupation for the game designers: not only have the ingame speeded (i.e. how fast your avatar is moving) but also the fluidity of the game, that depends notably on the data transfer technology. Massive efforts are done by the designers to prevent lags or delays in the game. Thus, the time correlation between potentially addictive stimuli and the reinforced cognitions and behaviors has been improved.

\section{CONCLUSIONS}

The aim of the present paper was to discuss similarities between Internet addiction and already established addictions. We have taken WoW as an illustration, comparing it notably with nicotine. It appears that various environmental stimuli which have been found to be associated with activations of the ventral striatum, are recurrently presented during the game, and may act as behavioral reinforcers leading eventually to addiction.

Regarding the first order vector aspects, it appears that the game regularly presents the salient stimuli in patterns corresponding to operant conditioning, using compound schedules optimizing on one hand the acquisition of the reinforced behavior, and on the other hand, its maintenance and its resistance to extinction.

Finally, the addictive properties of WoW have probably been facilitated by the last technological developments, rendering the game more accessible, and permitting very strong time correlations between potentially addictive stimuli and the reinforced cognitions and behaviors. 
In conclusion, the discussed similarities between WoW and nicotine addiction, support the claim of considering Internet addiction as a sub-category of addictive behaviors.

\section{ACKNOWLEDGEMENT}

Declared none.

\section{CONFLICT OF INTEREST}

Declared none.

\section{REFERENCES}

[1] Baldwin AE, Sadeghian K, Kelley AE. Appetitive instrumental learning requires coincident activation of NMDA and Dopamine D1 Receptors within the medial prefrontal cortex. J Neurosci 2002; 22(3): 1063-71.

[2] Walter H, Abler B, Ciaramidaro A, Erk S. Motivating forces of human actions. Neuroimaging reward and social interaction. Brain Res Bull 2005; 67(5): 368-81.

[3] Volkow ND, Li TK. Drug addiction: the neurobiology of behaviour gone awry. Nat Rev Neurosci 2004; 5(12): 963-70.

[4] Horvitz JC. Mesolimbocortical and nigrostriatal dopamine responses to salient non-reward events. Neurosci 2000; 96(4): 6516.

[5] Joseph MH, Datla K, Young AM. The interpretation of the measurement of nucleus accumbens dopamine by in vivo dialysis: the kick, the craving or the cognition? Neurosci Biobehav Rev 2003; 27(6): 527-41

[6] Cannon CM, Bseikri MR. Is dopamine required for natural reward? Physiol Behav 2004; 81(5): 741-8.

[7] Walther JB. Computer-mediated communication impersonal, interpersonal, and hyperpersonal interaction. Commun Res 1996; 23(1): 3-43

[8] Koepp MJ, Gunn RN, Lawrence AD, et al. Evidence for striatal dopamine release during a video game. Nature 1998; 393(6682): 266-8.
[9] Tabibnia G, Lieberman MD. Fairness and cooperation are rewarding: evidence from social cognitive neuroscience. Ann N Y Acad Sci 2007; 1118: 90-101.

[10] Izuma K, Saito DN, Sadato N. Processing of social and monetary rewards in the human striatum. Neuron 2008; 58(2): 284-94.

[11] Rilling J, Gutman D, Zeh T, Pagnoni G, Berns G, Kilts C. A neural basis for social cooperation. Neuron 2002; 35(2): 395-405.

[12] Fehr E, Camerer CF. Social neuroeconomics: the neural circuitry of social preferences. Trends Cogn Sci 2007; 11(10): 419-27.

[13] Ducheneaut N, Yee N, Nickell E, Moore RJ. 'Alone together?' exploring the social dynamics of massively multiplayer online games. In: ACM Conference on Human Factors in Computing Systems; 2006. April 22-27; Montreal, Canada. NY: ACM; 2006; pp. 407-16.

[14] Singer T, Seymour B, O'Doherty JP, Stephan KE, Dolan RJ, Frith CD. Empathic neural responses are modulated by the perceived fairness of others. Nature 2006; 439(7075): 466-9.

[15] Zink CF, Tong Y, Chen Q, Bassett DS, Stein JL, MeyerLindenberg A. Know your place: neural processing of social hierarchy in humans. Neuron 2008; 58(2): 273-83.

[16] Festinger L. A theory of social comparison processes. Hum Relat 1954; 7: 117-40.

[17] Erk S, Spitzer M, Wunderlich AP, Galley L, Walter H. Cultural objects modulate reward circuitry. Neuroreport 2002; 13(18): 2499503.

[18] Fliessbach K, Weber B, Trautner P, et al. Social comparison affects reward-related brain activity in the human ventral striatum. Science 2007; 318(5854): 1305-8.

[19] Mobbs D, Greicius MD, Abdel-Azim E, Menon V, Reiss AL. Humor modulates the mesolimbic reward centers. Neuron 2003 ; 40(5): 1041-8.

[20] Volkow ND, Fowler JS, Wang GJ, Swanson JM, Telang F. Dopamine in drug abuse and addiction: results of imaging studies and treatment implications. Arch Neurol 2007; 64(11): 1575-9.

This is an open access article licensed under the terms of the Creative Commons Attribution Non-Commercial License (http:/creativecommons.org/licenses/ by-nc/3.0/) which permits unrestricted, non-commercial use, distribution and reproduction in any medium, provided the work is properly cited. 